\title{
ZenTrader, an Emotion-Reactive Interface
}

\author{
Thomas Luan Dang, Steven Kun Liu, and Sidney Fels \\ University of British Columbia, 2329 West Mall, Vancouver, BC Canada V6T1Z4 \\ dqluan@gmail.com, skliu_ubc@hotmail.com, \\ ssfels@ece.ubc.ca
}

\begin{abstract}
We explore the concept of interactive technology that is implicitly controlled by emotions, via wearable physiology sensors. We describe a proofof-concept emotion-reactive stock trading software that interrupts trades that appear to be entered under an unusual amount of stress. We describe a galvanic skin response (GSR) sensor armband that broadcasts stress events via Bluetooth to a prisoner's dilemma game application on an Android phone, which alters its behavior when the user is stressed. We carried out pilot evaluation of this system's effects on the consistency of decisions made under stress.
\end{abstract}

Keywords: interactive technology, finance, GSR, EDA, stress, galvanic skin response, implicit control, wearable, affective computing, proactive computing.

\section{Introduction and Related Work}

The idea of computers that can anticipate our emotions and react accordingly, first envisioned in the early 2000s[17, 20], is potentially attractive in many domains, such as entertainment, education, health care, finance, and services. However, few such applications have emerged, due to various challenges[17]. Sensors of physiological metrics are complicated and difficult to deploy or wear outside of the lab. Emotions are difficult to predict from raw sensor data, especially when different models may be required for different human activities. Finally, the interfaces and interactions being implicitly controlled by human emotions may require different design considerations than the interactions controlled by explicit input.

To date, several emotion-reactive entertainment applications have been developed. StartleCam[5] is a camera that detects when its user is startled via electrodermal activities (EDA, aka. galvanic skin response), and snaps a picture. 2Hearts is a novel musical instrument that extracts variables from two people's heartbeats in order to create music[12]. Very recently, a system was developed that also used galvanic skin response to create bookmarks within audiobook tracks when the reader is interrupted by external stimuli[14]. In parallel, there have been some research on quantitatively modeling the emotions of people in specific activities based on physiological metrics, such as in learning[6] or during gameplay[10], which may allow future games to make more sophisticated and reliable predictions of players' emotions.

In this project, we explore the emotion-reactive concept in stock trading. Trading is rather dissimilar to entertainment, as it is result-oriented instead of experienceoriented, and yet also similar, as emotions, stress in particular, often play a critical role in determining results. On the market, the best opportunities or the signs of 
danger are often erratic and time-sensitive, yet the intention of trading is to produce profit consistently. As a result, a trader sitting in a desk for hours or weeks waiting for potentially important events can suffer from severe anxiety[13]. This anxiety often contributes to irrational, inconsistent behaviors[4], such as excessive risk taking or deviating from strategic and execution plans. Previous studies have monitored the physiology of traders [8, 9] and Poker players [19]. Results confirm that decisions with real financial stakes tend to cause stress, which manifests as measurable difference in physiological metrics, such as galvanic skin response, heart rate, and body temperature... The involvement of real financial stakes in emotion monitoring experiments is apparently critical in obtaining such results.

Passive sensing and display of stress level have been recently explored for trading, Panasonic[15] and Philips[16], have created wearable prototypes that incorporate both a GSR sensor and a display to visualize the traders' stress-level. For monitoring stress caused by exposure to addictive substances, the AutoSense system[18, 21] use wearable sensors to communicate wirelessly with a mobile display. However, all of these systems are passive displays of emotions, which could very well be ignored in the heat of the moment.

In this paper, we introduce ZenTrader, a trading system that tailors its interface and allowable actions to the trader's stress level. The system is implicitly controlled partly by the stress level to proactively warn against anxious behaviours. It can also suggest background tasks, such as market research or strategic planning, whenever the stress level is low. The net effect is to keep the trader in more of a "flow" state [3], where cognitive and emotional functions are at their peak. To date, there have been only a few such applications that use physiological sensors for implicit control, and there appear to be none in the finance domain.

In the context of related works on physiological sensing, wearable devices, and implicit control already discussed, our contributions to Interactive Technology are i) identifying that trading and other stress-sensitive activities do not need to have a wearable interface (instead of existing mobile interfaces), but the subconscious act of sensing moments of stress requires continuous, constant availability, and so benefits from wearability; ii) proposing that interfaces for stress-sensitive activities should be partly controlled implicitly by the stress level; iii) prototyping a wearable GSR sensor, which updates a stress-reactive application wirelessly.

\section{Conceptual Design and Proof-of-Concept Prototype}

In the mental model of a traditional computerized trading platform (Fig. 1), the trading software (computer) is static and passive, with an input facility. Even if the input facility can be as complicated as a script of trading strategies, instead of single trades, the computer is still a passive thrall to the will of the user. However, various infamous incidents in the financial industry, such as the Fake Bankruptcy of United Airline[11], suggest that the will of users may not always serve their best interest.

At any given moment, people output not only their rational thoughts, but also their emotions. ZenTrader attempts to respond to both the explicit and emotional inputs (Fig. 1). ZenTrader still consists of a trading application running on one or more devices. This application still takes explicit commands from its user. However, since 
emotions took a part in forming those commands, these emotions must also be an input into the application. Emotions are often subconscious, so they have to be sensed. A sensor worn by the trader autonomously and constantly measures various physiological metrics, interprets this data as shifts in stress levels, and informs the trading application. When a trade is entered while the stress level seems abnormally high, the trading application interrupts the command, forcing the trader to think twice or to type in a rationale. On the other hand, at moments where the user's stress level is so far below even a normal baseline, the system would suggest some trading, market research, or strategic planning activities.
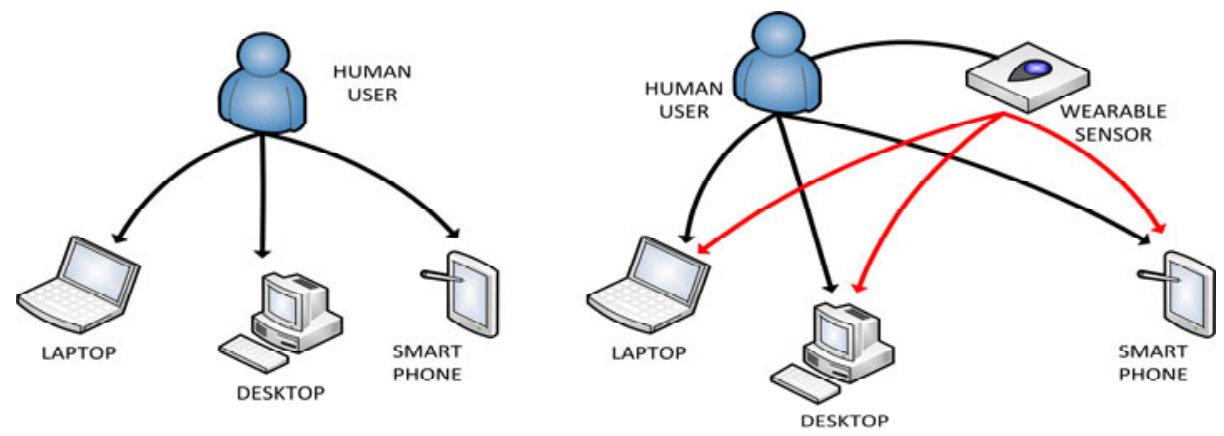

Fig. 1. Traditional Mental Model (left) vs. Emotion-Reactive Mental Model (right)

To evaluate the emotion-reactive concept, we simulate the trading application in our prototype with a noisy iterative prisoner's dilemma game to facilitate repeatable, controlled experiments. We realized that scientifically evaluating the effectiveness of the concept with real market condition is not practical for this stage of our research. Nevertheless, the low-fidelity prisoner's dilemma game prototype still contains all the elements of the ZenTrader mental model, such as the wearable GSR sensor, wireless broadcast, and a target application that receives and reacts to this broadcast. We originally planned to implement a GSR, skin temperature, and heart rate sensor, in order to triangulate the three data sources and produce a more reliable stress indicator. However, to simplify the prototype and keep the size and weight small, we decided to use only GSR. We developed our prisoner's dilemma application on the Java-based Android OS, and linked to the wearable GSR sensor via Bluetooth. We built our own GSR sensor armband instead of using an FDA-approved commercial solution, such as ThoughtTechnologies's ProComp[1] or BodyMedia's Sensewear[2], because we also seek to investigate the problems in tuning the form factor of a wearable GSR sensor.

The prototype form-factor consists of a wide, elastic armband to distribute the weight (roughly that of a cellphone) and create a secure grip. The armband is attached to the sensor unit housing with Velcro to facilitate testing with different wearing position and orientation. During development, the GSR electrodes were not attached under the armband (Fig. 2), but pressed into the skin by another elastic armband, so that the location of the sensor and the distance between the electrodes could be tuned. In the final design, the electrodes are secured to the main armband and pressed tightly against the palmer (under) side of the wrist. 
The GSR sensor circuit is built on a plain prototype board. The GSR sensor data is fed serially to an Arduino Uno board, with a Bluetooth Mate modem that acts as a wireless serial pipe to send signal from our Arduino-board-mounted sensors to the Android target application. We developed our target application using Amarino, an open source (LGPL) library that allows Arduino to send and receive data to/from an Android device[7]. The signal processing and stress event recognition logic are built into the target application, not the sensor unit, because this logic is application / domain-specific. We first take a 3-minute baseline of the user's GSR when he/she is calm each time the sensor is worn. The Android-based application will collect the data into a buffer of four seconds, which is compared to the baseline when full.

When the user chooses Defect or Cooperate each round, if the difference between the baseline and the moving average GSR data exceeds a predetermined threshold, a stress warning will appear in the top text box (Fig. 3), and the phone will vibrate, forcing the user to choose (think) twice per round.

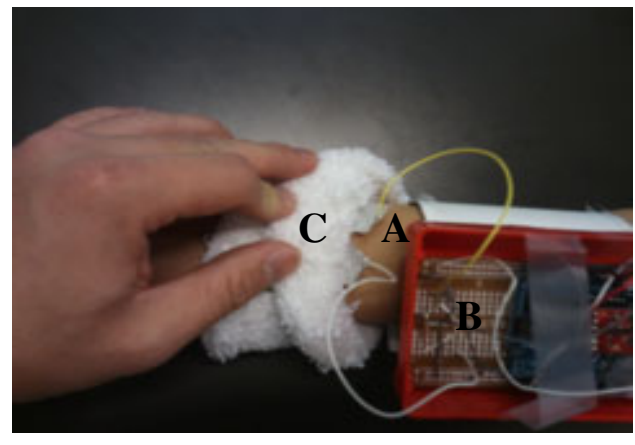

Fig. 2. (Left) Low-Fidelity prototype form factor. (A) The electrodes (aluminum) are detached from the main armband and circuitry (B) for position tuning using another elastic armband (C).

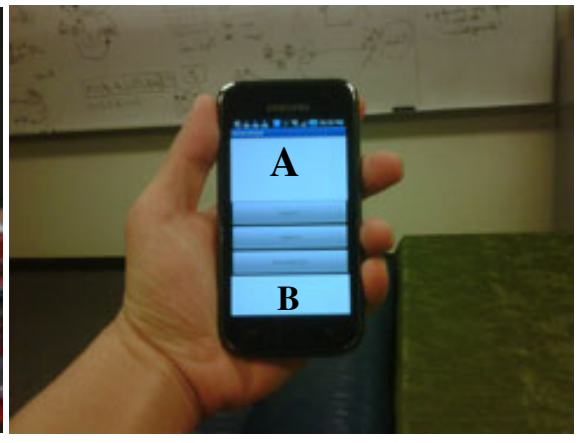

Fig. 3. (Right) Android phone with sample application (prisoner's dilemma). (A) Top Text Box: game text (pay-off matrix) and stress warning, Top Button:

Defect, Middle Button: Cooperate, Bottom Button: Create Baseline, (B) Bottom Box: skin resistance graph.

We tuned a threshold in the prototype so that the system would identify a stress event within 10-15 seconds of an unexpected, strong, and persistent stimulus, such as very loud music. The baseline data and the on-going data are buffered and averaged to reduce the responsiveness of the sensor enough that it will not respond to startle or orienting responses, which are immediate reflexive behaviors to short-term stimuli, like a tap from behind. For trading and game applications, the sensor should identify moments when there are persistent responses in skin conductance, which indicate ongoing changes in arousal level. More importantly, tuning the system to ignore shortterm involuntary reflexes is crucial for wearability in daily life. The GSR sensor in our prototype indicates "arousal", not any well-defined state of emotion, but persistent arousal is a good initial estimate for stress in trading, because extreme, on-going emotions, either greed or fear, can cause stress. Creating a more rigorous quantitative 
model of stress specifically for trading environments is out of the scope of our work at this stage, but this work paves the way for more field testing that will enable the construction of such a model.

\section{Pilot Evaluation}

We ask the research question: "At the point of decision-making, do interrupting warnings about stress induce more consistent, systematic behaviours?" We chose to conduct a small-scale pilot evaluation of the system, using a prisoner's dilemma (PD) game task that provides an analog to trading. The subjects play fast-paced 3-player iterative PD games. The control subjects use a normal version of the game that is not stress-aware, and the experimental subjects wear the sensor and use the emotionreactive version of the game. The pay-off matrix is procedurally tweaked after certain random number of rounds, so that the optimal strategy changes between sets of rounds. The experiment measures changes of strategy between sets of trials, identifiable by who wins each set, and also by the ratio of Defects and Cooperate. We obtained some promising results from the pilot experiment. The subjects using the device seemed to be more consistent with their first-set strategy, regardless whether this strategy was still favourable later.

\section{Conclusions}

In this paper, we explored the concept of applications that actively respond to human subconscious input by the use of continuously available wearable sensors. We described the mental model for such an emotion-reactive trading system, and built a proof-of-concept prototype involving a prisoner's dilemma game. We also carried out a small pilot evaluation, finding initial promising results. We believe that there are many challenges in the design, implementation, and adoption of emotion-reactive systems, but the challenges can be overcome. As physiological sensors become more reliable, more wearable, and more fashionable, the ubiquity and social acceptance of wearable sensors would increase. As API's that connect sensors and devices, such as Amarino, become more mature and available on more platforms, it will be easier to implement sensors with wireless broadcast.

Our work has several limitations, which we intend to address in future projects. Only a small pilot evaluation was conducted, limiting the validity of the results. A more robust methodology consideration and a more extensive experiment are needed. The armband sensor, though relatively light and wearable for a prototype, is still quite unwieldy for practical use. We believe that it is possible to miniaturize and house this sensor unit in a wrist-watch-sized enclosure, by printing the entire GSR sensor circuit on one chip. Finally, we believe that a longitudinal field study of traders, with real financial stakes, would be needed to identify real-world issues.

Acknowledgments. We would like to extend a sincere thanks to Dr. Ronald Rensink, Dr. Joanna McGrenere, Dr. Karon Maclean, and Thomas's former colleague William Ono for giving us helpful suggestions and proof-reading this paper. 


\section{References}

1. Thought Technology Ltd.: Biofeedback, Neurofeedback, Muscle Rehabilitation Equipment \& Services, http: / / www . thoughttechnology . com

2. Body Media, I.: Assessment of Physical Activity Using a Multi-sensor Armband. In: European Respiratory Society Meeting, Munich, Germany (2006)

3. Czikszentmihalyi, M.: Flow: The psychology of optimal experience, 1st edn. Harper Perennial (1991)

4. Haugen, R.: The Inefficient Market and the Potential Contribution of Behavioral Finance: Case Closed. In: CFA Institute Conference Proceedings Quarterly, pp. 6-14 (2010)

5. Healey, J., Picard, R.W.: StartleCam: A Cybernetic Wearable Camera. In: The International Symposium on Wearable Computers, Pittsburgh, PA, USA (1998)

6. Kapoor, A., Burleson, W., Picard, R.W.: Automatic Prediction of Frustration. Int. J. Hum.Comput. St. 65, 724-736 (2007)

7. Kaufmann, B.: Amarino - "Android Meets Arduino". 2.0 (2010)

8. Lo, A.W., Dmitry, R.V.: The Psychophysiology of Real-Time Financial Risk Processing. J. Cognitive Neurosci. 14, 323-339 (2002)

9. Lo, A.W., Dmitry, R.V., Steenbarger, B.N.: Fear and Greed in Financial Markets: A Clinical Study of Day-Traders. Am. Econ. Rev. 95, 352-359 (1995)

10. Mandryk, R.L., Atkins, M.S., Inkpen, K.M.: A Continuous and Objective Evaluation of Emotional Experience with Interactive Play Environments. In: The Conference on Human Factors in Computing Systems (CHI 2006), Montreal, Canada, pp. 1027-1036 (2006)

11. Maynard, M.: United Shares Drop on False Bankruptcy Report, NY Times (2008)

12. McCaig, G., Fels, S.: Playing on Heart-Strings: Experience with the 2Hearts System. In: The 2002 Conference on New Interfaces for Musical Expression (NIME 2002), pp. 1-6 (2002)

13. Osuna, E.E.: The Psychological Cost of Waiting. J. Math. Psycho. 29, 82-105 (1985)

14. Pan, M.K.X.J., Chang, J.S., Himmetoglu, G.H., Moon, A., Hazelton, T.W., Maclean, K.E., Croft, E.A.: Now, Where Was I? Physiologically Triggered Bookmarks for Audio Books. In: ACM Conference on Human Factors in Computing Systems, CHI 2011 (2011)

15. Panasonic, I.: The Emo Bracelet (2007)

16. Philips, I.: Emo Bracelet and Emo Bowl (2009)

17. Picard, R.W.: Toward Machines with Emotional Intelligence. In: Matthews, G., Zeidner, M., Roberts, R.D. (eds.) The Science of Emotional Intelligence: Knowns and Unknowns. Oxford University Press, Oxford (2007)

18. Sung, M.: Non-Invasive Wearable Sensing Systems for Continuous Health Monitoring and Long-Term Behavior Modelling. Ph. D. Thesis (2005)

19. Sung, M., Pentland, A.: Pokermetrics: Stress and Lie Detection Through Non-invasive Psychological Sensing (2005)

20. Tennenhouse, D.: Proactive Computing. Commun. ACM 43 (2000)

21. AutoSense: http://sites.google.com/site/autosenseproject/home 